\title{
TractoFlow-ABS (Atlas-Based Segmentation)
}

\author{
Guillaume Theaud $^{\mathrm{a}, \mathrm{b}, *}$, Jean-Christophe Houde ${ }^{\mathrm{a}, \mathrm{b}}$, Arnaud Boré ${ }^{\mathrm{a}}$, François Rheault ${ }^{\mathrm{a}}$ \\ Felix Morency $^{\mathrm{b}}$, Maxime Descoteaux ${ }^{\mathrm{a}, \mathrm{b}}$ \\ ${ }^{a}$ Sherbrooke Connectivity Imaging Laboratory (SCIL), Université de Sherbrooke, Canada \\ ${ }^{b}$ Imeka Solutions Inc, Sherbrooke, Canada
}

\begin{abstract}
In Diffusion MRI (dMRI), pathological brains are a challenge for tractography processing, where most pipelines are not are not robust to white matter lesions. Intensity of white matter lesions on T1 images can have similar contrasts to gray matter tissue, which leads to misclassifications or "holes" in the white matter mask. These holes produce premature stop for tracking algorithms. To handle these issues, we developed TractoFlow-ABS (Atlas-Based Segmentation). TractoFlow-ABS uses the Freesurfer atlas to compute tissue masks instead of FSL fast in standard TractoFlow. TractoFlow-ABS is therefore a derived version of TractoFlow that is robust to white matter anomalies such as hyperintensities and lesions.
\end{abstract}

\section{Introduction}

White matter can be affected by multiple types of lesions due to aging and multiple sclerosis, amongst other diseases. In the field of diffusion MRI, these lesions can affect DWI processing and more precisely the tractography step [Edde et al., 2019; Theaud et al., 2017]. In order to do the best processing as possible, a version of TractoFlow [Theaud et al., 2020] was developed for brains where the usual T1 segmentation step fails. Compared to TractoFlow, TractoFlow-ABS uses Freesurfer to compute tissue masks instead of fast from FSL. Lesion instensities on T1 images are similar to gray matter tissue, which leads to misclassifications from fast. Using Freesurfer allows to have a better tissue classification, which then results in better tracking masks and a better tractogram. Otherwise, the tractograpy process stops prematureraly due the holes and the misclassified gray matter voxels in the deep white matter.

\section{Methods}

TractoFlow-ABS is based on TractoFlow [Theaud et al., 2020].

\footnotetext{
*2500, boul. de l'Université, Sherbrooke (Québec) Canada, J1K 2R1

Email address: Guillaume. Theaud@usherbrooke.ca (Guillaume Theaud)
} 
bioRxiv preprint doi: https://doi.org/10.1101/2020.08.03.197384; this version posted August 4, 2020. The copyright holder for this preprint (which was not certified by peer review) is the author/funder, who has granted bioRxiv a license to display the preprint in perpetuity. It is made available under aCC-BY-NC-ND 4.0 International license.
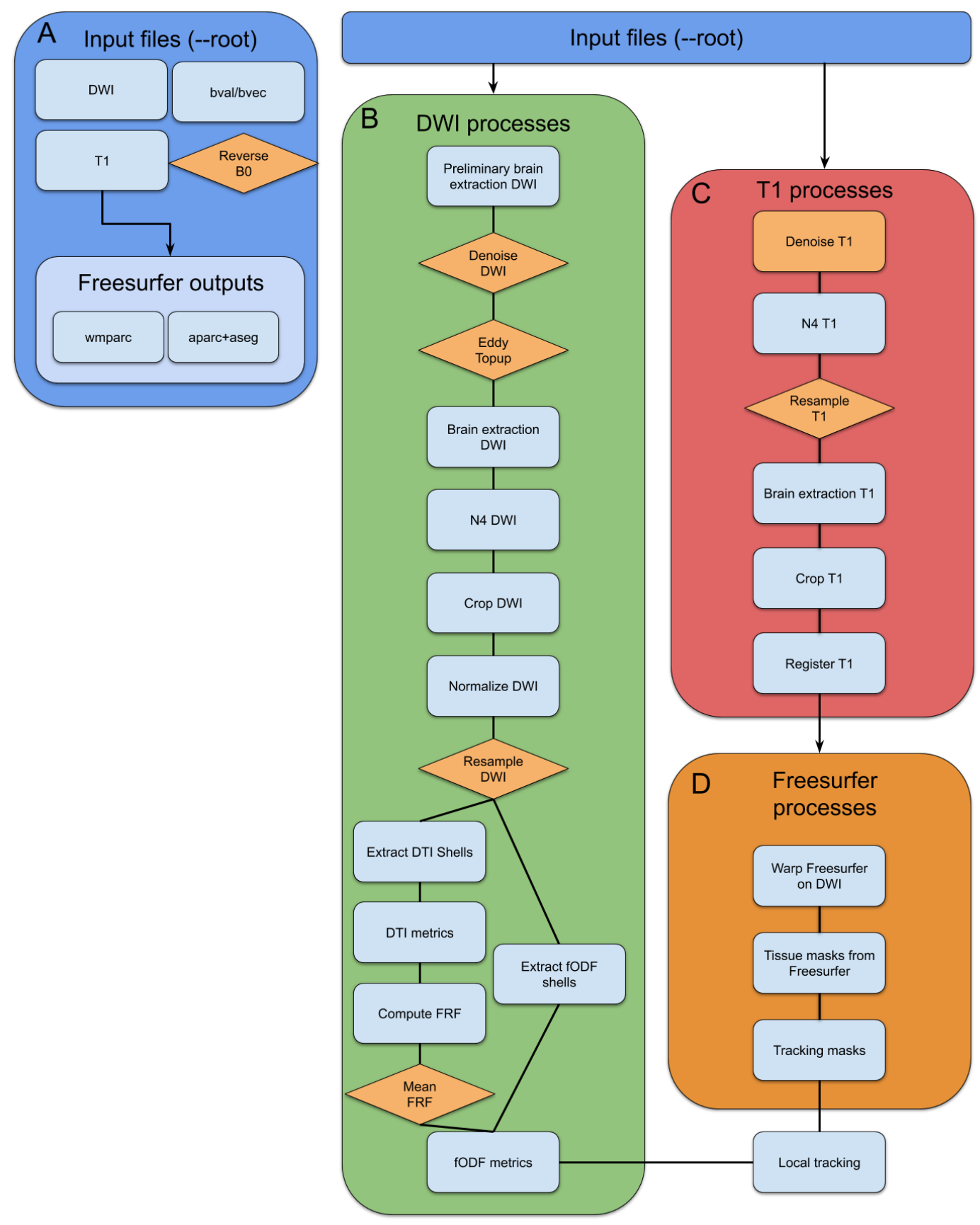

Figure 1: The graph of processes of the TractoFlow-ABS pipeline. In A (blue), the input files required to run the pipeline. In B (green), the DWI processes that take the DWI, the b-values/b-vectors files and the reversed phase encoded $\mathrm{b}=0$ image. In $\mathrm{C}(\mathrm{red})$, the $\mathrm{T} 1$ processes that take the T1-weighted image as input. In D, the Freesurfer processes that take wmparc and aparc+aseg. In orange, all processes or images that are optional. 

made available under aCC-BY-NC-ND 4.0 International license.

Pipeline inputs. TractoFlow-ABS requires a Freesurfer [Fischl, 2012] output computed from the original $\mathrm{T} 1$ in native space. Then, the pipeline takes as input: the diffusion weighted images, T1 weighted image, b-values and b-vectors, wmparc and aparc + aseg from Freesurfer. As in TractoFlow, if available, the pipeline accepts a reversed phase encoded $b=0$ image. These input files are illustrated in Figure 1.A.

Diffusion-weighted images (DWI) tasks. The DWI tasks are the same as presented in the original TractoFlow paper [Theaud et al., 2020]. The steps are illustrated in Figure 1.B.

T1-weighted image tasks. T1-weighted image tasks are similar to TractoFlow except for the tissue maps and the tracking maps steps, as seen in 1.C. As said in the introduction, with aging or multiple sclerosis, white matter lesions appear and can impact results of tractography algorithms by creating holes in the tracking mask. In order to fill in the holes due to white matter lesions, some Freesurfer tasks are added to TractoFlow-ABS. It is important to point out that one could decide to use this strategy for healthy brains.

Freesurfer tasks. Freesurfer tasks consist of 3 steps (1.D). First, Freesurfer wmparc and aparc + aseg output files are warped on the DWI using the nonlinear transformation from the T1-to-Diffusion registration step, with nearestneighbor interpolation. Then, WM (white matter), GM (gray matter), CSF (cerebral spinal fluid) and nucleii masks are extracted from the Freesurfer images combining the appropriate labels (see Figure 2). Finally, tracking and seeding masks are created.

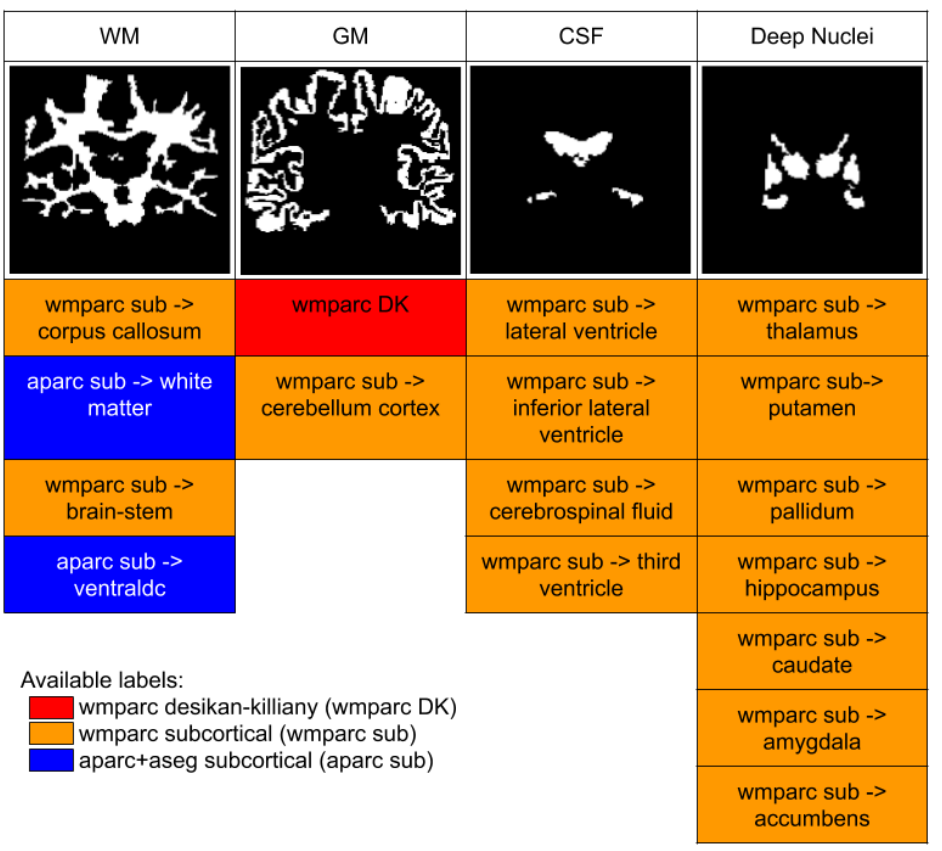

Figure 2: Details of Freesurfer label combining. 
bioRxiv preprint doi: https://doi org/10.1101/2020.08.03 197384 this version posted August 4, 2020. The copyright holder for this preprint (which was not certified by peer review) is the author/funder, who has granted bioRxiv a license to display the preprint in perpetuity. It is made available under aCC-BY-NC-ND 4.0 International license.

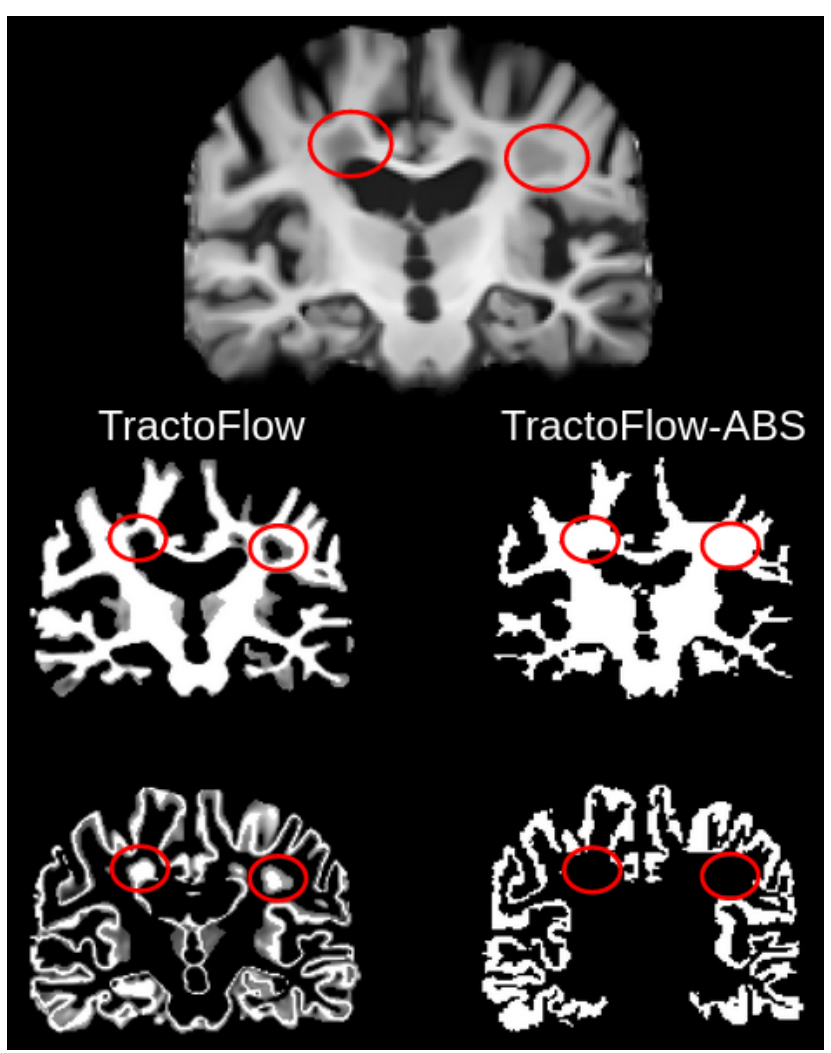

Figure 3: In red circles, lesions locations. In the left column, tissue maps results from TractoFlow. In the right column, tissue maps from TractoFlow- $A B S$

Tracking task. For TractoFlow-ABS, the tracking algorithm used is the local tracking [Descoteaux et al., 2009], as opposed to the particle filter tractography (PFT) algorithm used in TractoFlow, which requires probabilistic tissue maps. As in TractoFlow, step size, number of seeds per voxel, deterministic or probabilistic algorithms can be selected by the user with command-line options.

\section{Results}

In this part, TractoFlow and TractoFlow-ABS are compared on a simple illustrative example of tissue maps and tractography output of the two pipelines using a subject from the ADNI database.

Tissue maps comparisons. Tissue maps segmented from TractoFlow are impacted by the WM lesions. Lesions are classified as GM instead of WM, as seen in Figure 3. This misclassification has a big impact on tracking masks, which are derived from theses tissue maps. Impact on tractography will be seen in the next section.

With TractoFlow-ABS, this misclassification is solved by the freesurfer atlas, and the WM lesions are classified as WM and not GM. This improvement will permit the 
bioRxiv preprint doi: https://doi.org/10.1101/2020.08.03.197384; this version posted August 4, 2020. The copyright holder for this preprint (which was not certified by peer review) is the author/funder, who has granted bioRxiv a license to display the preprint in perpetuity. It is made available under aCC-BY-NC-ND 4.0 International license.

tractography algorithm to explore this region and reconstruct pathways through these lesions, if coherent local orientations are present (as seen in Figure 5).

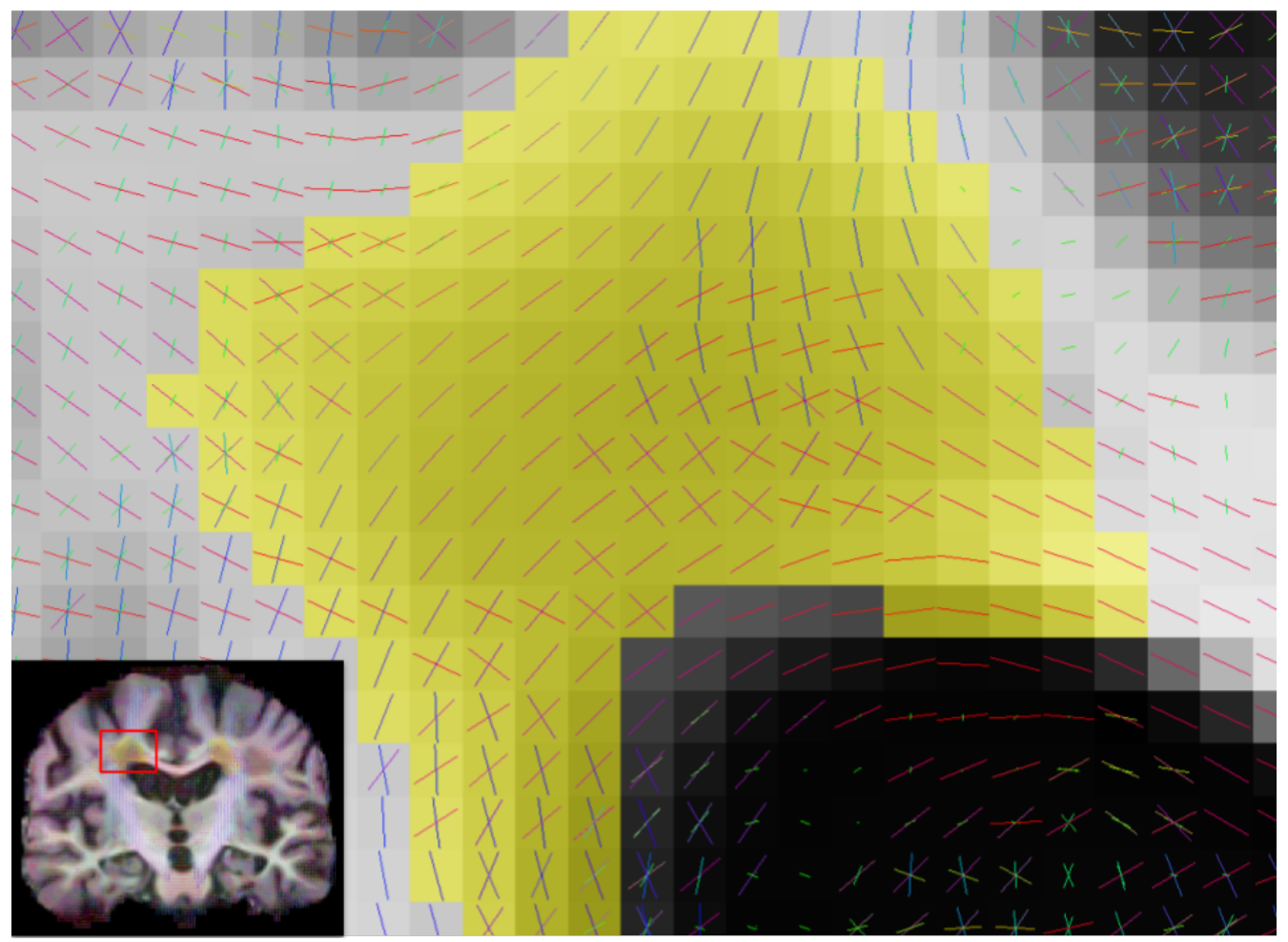

Figure 4: In yellow, a white matter lesion. Local orientations in the lesions are coherent. Local tractography can thus reconstruct pathway through this lesion.

Tractography comparisons. As previously mentioned, if holes are present in tracking masks, tractography algorithms cannot track in this region. With TractoFlow, the lesions create GM voxels in the middle of the deep WM, which makes the tracking algorithm i) allowed to stop because there are falsely labeled GM voxels, and ii) prevents the tracking algorithm to explore through that region of WM. For example, Figure 5 shows a part of the corpus callosum segmented from the TractoFlow and TractoFlow-ABS tractogram. The extracted corpus callosum from TractoFlow contains broken streamlines by the lesions and lacks a large number of commissural streamlines, reconstructed by TractoFlow- $A B S$, going to the lateral left and right cortices. 
bioRxiv preprint doi: https://doi.org/10.1101/2020.08.03 197384; this version posted August 4, 2020. The copyright holder for this preprint (which was not certified by peer review) is the author/funder, who has granted bioRxiv a license to display the preprint in perpetuity. It is made available under aCC-BY-NC-ND 4.0 International license.

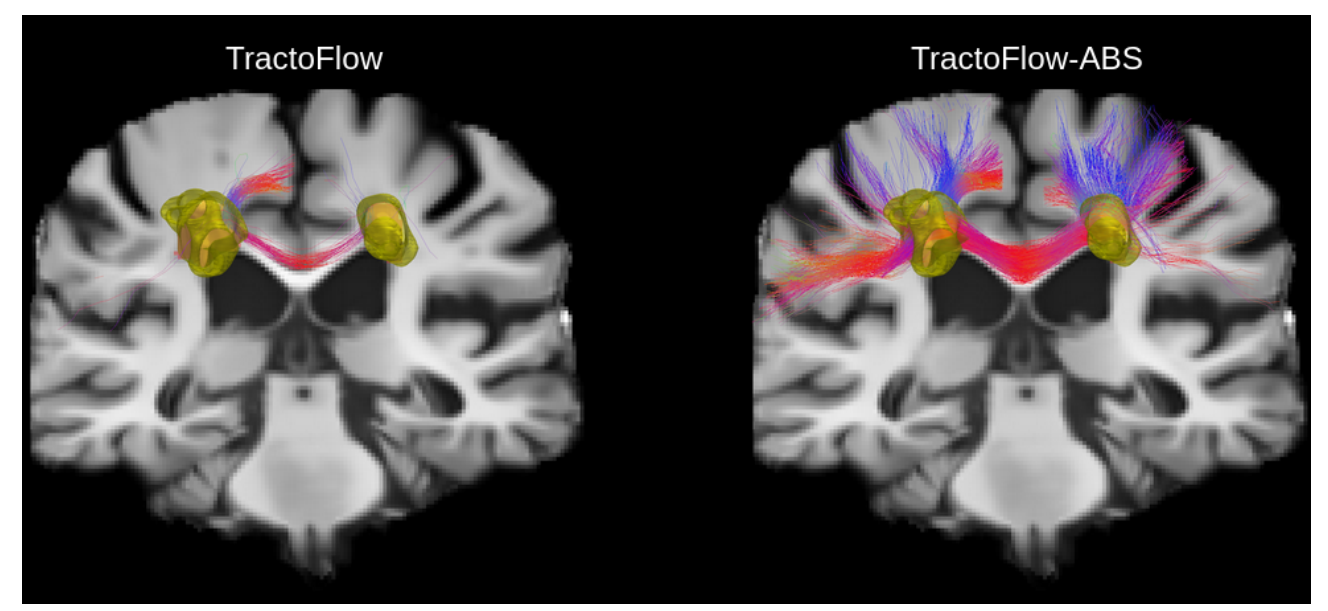

Figure 5: The corpus callosum extracted from TractoFlow and TractoFlow-ABS tractograms showed in a coronal slice view. In yellow, WM lesions are shown.

\section{Conclusion}

TractoFlow-ABS uses the Freesurfer atlas to compute its tracking mask. As a result, in datasets with WM lesions, it is able to reconstruct a better WM mask, which allows tractography to track through the WM lesions if coherent local orientations exist. This improvement is thus mostly due to better tissue maps that come from the Freesurfer atlas. It is important to say that if one prefers the Freesurfer white matter segmentation than the FSL fast segmentation, it is possible to run this pipeline on healhty brains as well. TractoFlow-ABS is available on Github under license (https://github.com/ scilus/TractoFlow-ABS).

\section{References}

Descoteaux, M., Deriche, R., Knosche, T.R., Anwander, A., 2009. Deterministic and probabilistic tractography based on complex fibre orientation distributions. IEEE transactions on medical imaging $28,269-286$.

Edde, M., Theaud, G., Rheault, F., Dilharreguy, B., Helmer, C., Dartigues, J.F., Amieva, H., Allard, M., Descoteaux, M., Catheline, G., 2019. Free water: a sensitive metric for age-related modifications of the cingulum white matter and its association with cognition. bioRxiv, 867606 .

Fischl, B., 2012. Freesurfer. Neuroimage 62, 774-781.

Theaud, G., Dilharreguy, B., Catheline, G., Descotaux, M., 2017. Impact of white-matter hyperintensities on tractography, in: 25th Annual Meeting of the International Society for Magnetic Resonance in Medicine (ISMRM). Honolulu: International Society for Magnetic Resonance in Medicine.

Theaud, G., Houde, J.C., Boré, A., Rheault, F., Morency, F., Descoteaux, M., 2020. Tractoflow: A robust, efficient and reproducible diffusion mri pipeline leveraging nextflow \& singularity. NeuroImage 116889. 